\title{
Multiplicity of Solutions for Neumann Problems for Semilinear Elliptic Equations
}

\author{
Yu-Cheng An ${ }^{1}$ and Hong-Min Suo ${ }^{2}$ \\ ${ }^{1}$ School of Sciences, Bijie University, Bijie 551700, China \\ ${ }^{2}$ School of Sciences, Guizhou Minzu University, Guiyang 550025, China \\ Correspondence should be addressed to Yu-Cheng An; anyucheng@126.com
}

Received 19 February 2014; Accepted 18 May 2014; Published 29 May 2014

Academic Editor: Leszek Gasinski

Copyright (c) 2014 Y.-C. An and H.-M. Suo. This is an open access article distributed under the Creative Commons Attribution License, which permits unrestricted use, distribution, and reproduction in any medium, provided the original work is properly cited.

Using the minimax methods in critical point theory, we study the multiplicity of solutions for a class of Neumann problems in the case near resonance. The results improve and generalize some of the corresponding existing results.

\section{Introduction}

The aim of this paper is to study the following semilinear Neumann problem:

$$
\begin{aligned}
-\Delta u+\beta(x) u & =\lambda u+f(x, u), \quad x \in \Omega, \\
\frac{\partial u}{\partial n} & =0, \quad x \in \partial \Omega .
\end{aligned}
$$

Here $\Omega \subset R^{N}(N \geq 3)$ is a bounded domain with a $C^{1}$ boundary $\partial \Omega$ and $n(\cdot)$ denotes the outward unit normal on $\partial \Omega$. Also $\beta \in L^{s}(\Omega)$ with $s>N / 2$ and it may change sign. The reaction $f(x, u)$ is a Carathéodory function and satisfies the following assumptions:

$\left(f_{0}\right)$ for every $M>0$, there exists a function $L_{M} \in L^{2}(\Omega)$ such that $|f(x, t)| \leq L_{M}(x)$ for all $|t| \leq M$ and a.e. $x \in \Omega$;

$$
\left(f_{\infty}\right) \lim _{|t| \rightarrow \infty}(f(x, t) / t)=0 \text { uniformly for } x \in \Omega \text {. }
$$

Recently, there have been many papers concerned with the Neumann problems; see [1-5] and the references therein. Or, more specifically, in Li [1] and Qian [2], the left hand side differential operator is $-\Delta u+\beta u$, with $\beta \in R$, $\beta>0$. In Motreanu et al. [3], Tang and Wu [4], and Motreanu et al. [5], the differential operator is $-\Delta u$ (i.e., $\beta=0$ ). Semilinear Neumann problems with unbounded and indefinite potential, especially, were studied by Gasiński and
Papageorgiou [6]. They obtained two multiplicity theorems. In addition, the same problems were studied by Papageorgiou and Rădulescu [7]. They dealt with equations in which the reaction $f(x, u)$ exhibits an asymmetric behavior at $+\infty$ and at $-\infty$ (jumping nonlinearity) and they proved multiplicity theorems providing sign information for all the solutions.

On the other hand, for the perturbed problem, Mawhin and Schmitt [8] first considered the two-point boundary value problem

$$
-u^{\prime \prime}-\lambda u=f(x, u)+h(x), \quad u(0)=u(\pi)=0 .
$$

Under the assumption that $f$ is bounded and satisfies a sign condition, if the parameter $\lambda$ is sufficiently close to $\lambda_{1}$ from left, problem (2) has at least three solutions; if $\lambda_{1} \leq \lambda<\lambda_{2}$, problem (2) has at least one solution, where $\lambda_{1}, \lambda_{2}$ are the first and second eigenvalues of the corresponding linear problem. Ma et al. [9] considered the boundary value problem $\Delta u+$ $\lambda u+f(x, u)=h(x)$ defined on a bounded open set $\Omega \subset R^{N}$, no matter whether the boundary conditions are Dirichlet or Neumann condition; as the parameter $\lambda$ approaches $\lambda_{1}$ from left, there exist three solutions. Moreover, existence of three solutions was obtained for the quasilinear problem in bounded domains as the parameter $\lambda$ approaches $\lambda_{1}$ from left. In $[10,11]$, these results were extended to the perturbed $p$-Laplacian equation in $R^{N}$. In [12], Ou and Tang extended above some results to some elliptic systems with the Dirichlet boundary conditions. de Paiva and Massa in [13], especially, 
studied the semilinear elliptic boundary value problem in any spatial dimension and using variational techniques; they showed that a suitable perturbation will turn the almost resonant situation $\left(\lambda\right.$ near to $\lambda_{k}$, i.e., near resonance with a nonprincipal eigenvalue) in a situation where the solutions are at least two. In [14], those results were extended to the degenerate elliptic equations in the bounded domain.

Motivated by the above idea, we have the goal in this paper of extending these results in $[6,12-14]$ to some elliptic equations with the Neumann boundary conditions. Here, it is worth pointing out that $\left(f_{\infty}\right)$ is weaker than $\left(f_{1}\right)$ in [13] (or $(A)$ in [14]). More to the point, there are functions satisfying the assumptions of our main results in Section 2 and not satisfying the assumptions in $[13,14]$. For example, let $f(x, t)=t / \ln (1+|t|)$. Then $f$ satisfies the assumptions for our Theorem 5 in Section 2 and does not satisfy $\left(f_{1}\right)$ in [13] (or $(A)$ in [14]).

The rest of our paper is organized as follows. In Section 2 we give some preliminary lemmas and our main results. Section 3 gives the detailed proofs of our main results based on several estimates, whose proof will be presented in Sections 4 and 5.

\section{Preliminaries and Main Results}

Let the Sobolev space $X=H^{1}(\Omega)$. Denote

$$
\|u\|_{1,2}=\left(\int_{\Omega}\left(|\nabla u|^{2}+u^{2}\right) d x\right)^{1 / 2}
$$

to be the norm of $u$ in $X$ and $\|u\|_{p}$ the norm of $u$ in $L^{p}(\Omega)$. The space $X$ is a Hilbert space. For a discussion about the space setting, we refer to [15] and the references therein.

Again, we recall the properties of the eigenvalue problem as follows (see [6]):

$$
\begin{gathered}
-\Delta u+\beta(x) u=\lambda u, \quad x \in \Omega, \\
\frac{\partial u}{\partial n}=0, \quad x \in \partial \Omega .
\end{gathered}
$$

The eigenvalue problems in (4) have a sequence $\left\{\lambda_{k}\right\}_{k \geq 1}$ of eigenvalues, such that

$$
\begin{array}{r}
-\infty<\lambda_{1}<\lambda_{2} \leq \lambda_{3} \leq \cdots \leq \lambda_{k} \leq \cdots \\
\text { as } k \longrightarrow+\infty
\end{array}
$$

There is also a corresponding sequence $\left\{\phi_{k}\right\}_{k \geq 1} \subset H^{1}(\Omega)$ of eigenfunctions which form an orthonormal basis of $L^{2}(\Omega)$ and an orthogonal basis of $H^{1}(\Omega)$. Moreover, we know that $\phi_{k} \in C^{1, \alpha}(\Omega)$ for some $\alpha \in(0,1)$ and all $k \geq 1$.

We denote by $E_{k}$ the eigenspace corresponding to an eigenvalue $\lambda_{k}$, and we can decompose $H^{1}(\Omega)=\bigoplus_{k=1}^{\infty} E_{k}$. We set

$$
\sigma(u)=\int_{\Omega}\left(|\nabla u|^{2}+\beta(x) u^{2}\right) d x .
$$

The eigenvalues admit the following variational characterizations in terms of the Rayleigh quotient $\sigma(u) /\|u\|_{2}^{2}$ for all $u \in H^{1}(\Omega)$ :

$$
\begin{aligned}
\lambda_{1} & =\inf \left\{\frac{\sigma(u)}{\|u\|_{2}^{2}}: u \in H^{1}(\Omega) \backslash\{0\}\right\}, \\
\lambda_{k} & =\inf \left\{\frac{\sigma(u)}{\|u\|_{2}^{2}}: u \in \bigoplus_{i=k}^{\infty} E_{i}, u \neq 0\right\} \\
& =\sup \left\{\frac{\sigma(u)}{\|u\|_{2}^{2}}: u \in \bigoplus_{i=1}^{k} E_{i}, u \neq 0\right\} .
\end{aligned}
$$

In (7), the infimum is realized on $E_{1}$. Also, in (8), both the infimum and the supremum are realized on $E_{k}$. All the eigenspaces have the so-called unique continuation property. The first eigenvalue $\lambda_{1}$ is simple and it is clear from (7) that the corresponding eigenfunctions do not change sign. Namely, we can suppose that $\phi_{1}$ is strictly positive on $\Omega$. We mention that all the other eigenvalues have nodal eigenfunctions. For more properties to the eigenvalue problem $(4)$, see $[6,7]$.

By the presence of function $\beta$, weak solutions of (1) must be found in a suitable space. To this purpose, letting $\theta>$ $\max \left\{-\lambda_{1}, 0\right\}$, we introduce a new inner product on $H^{1}(\Omega)$ by

$$
\langle u, v\rangle=\int_{\Omega}(\nabla u \nabla v+\beta(x) u v+\theta u v) d x
$$

for $u, v \in H^{1}(\Omega)$ and the associated norm

$$
\|u\|=\left(\int_{\Omega}\left(|\nabla u|^{2}+\beta(x) u^{2}+\theta u^{2}\right) d x\right)^{1 / 2}, \quad u \in H^{1}(\Omega) .
$$

Lemma 1. Let $\beta \in L^{s}(\Omega)$ with $s>N / 2$ and it may change sign, if $\theta>\max \left\{-\lambda_{1}, 0\right\}$; then $\|\cdot\|$ is equivalent to the usual Sobolev norm $\|\cdot\|_{1,2}$.

Proof. By virtue of Hölder's inequality, we have

$$
\begin{aligned}
& \int_{\Omega}\left(|\nabla u|^{2}+\beta(x) u^{2}+\theta u^{2}\right) d x \\
& \quad \leq \int_{\Omega}\left(|\nabla u|^{2}+\theta u^{2}\right) d x+\|\beta\|_{s}\|u\|_{2 s^{\prime}}^{2} \\
& \quad \leq \max \{1, \theta\}\|u\|_{1,2}^{2}+\|\beta\|_{s}\|u\|_{2 s^{\prime}}^{2} \\
& \quad \leq \max \{1, \theta\}\|u\|_{1,2}^{2}+K^{2}\|\beta\|_{s}\|u\|_{1,2}^{2} \\
& \quad \leq \xi_{2}\|u\|_{1,2}^{2},
\end{aligned}
$$

where $K$ is the constant of Sobolev imbedding from $H^{1}(\Omega) \hookrightarrow L^{2 s^{\prime}}(\Omega), s^{\prime}=s /(s-1), \xi_{2}=\max \left\{1, \theta, K^{2}\|\beta\|_{s}\right\}$.

On the other hand, if $\theta>\max \left\{-\lambda_{1}, 0\right\}$, then there exists $\xi_{1}>0$ such that

$$
\int_{\Omega}\left(|\nabla u|^{2}+\beta(x) u^{2}+\theta u^{2}\right) d x \geq \xi_{1}\|u\|_{1,2}^{2}
$$


If not, it is clear from (7) that

$$
\chi(u)=\int_{\Omega}\left(|\nabla u|^{2}+\beta(x) u^{2}+\theta u^{2}\right) d x \geq 0 .
$$

Exploiting the 2-homogeneity of $\chi$ we can find a sequence $\left\{u_{n}\right\}_{n \geq 1} \subset H^{1}(\Omega)$, such that

$$
\left\|u_{n}\right\|_{1,2}=1, \quad \forall n \geq 1, \quad \chi\left(u_{n}\right) \longrightarrow 0^{+} .
$$

Passing to a subsequence if necessary, we may assume that

$$
\begin{gathered}
u_{n} \rightarrow u \quad \text { weakly in } H^{1}(\Omega), \\
u_{n} \longrightarrow u \quad \text { strongly in } L^{2 s^{\prime}}(\Omega) .
\end{gathered}
$$

The sequential weak lower semicontinuity of $\sigma$ and (15) imply that

$$
\sigma(u) \leq-\int_{\Omega} \theta u^{2} d x \leq \lambda_{1}\|u\|_{2}^{2}
$$

So $\sigma(u)=\lambda_{1}\|u\|_{2}^{2}$ and thus $u=\gamma \phi_{1}$, with some $\gamma \in R$.

If $\gamma=0$, then $u_{n} \longrightarrow 0$ in $H^{1}(\Omega)$, which contradicts the fact that $\left\|u_{n}\right\|_{1,2}=1$ for all $n \geq 1$. If $\gamma \neq 0$, then, from (17), we have $\sigma\left(\phi_{1}\right)<\lambda_{1}\left\|\phi_{1}\right\|_{2}^{2}$, which contradicts (7).

Combining (11) and (12), we have

$$
\xi_{1}\|u\|_{1,2}^{2} \leq \int_{\Omega}\left(|\nabla u|^{2}+\beta(x) u^{2}+\theta u^{2}\right) d x \leq \xi_{2}\|u\|_{1,2}^{2}
$$

Namely, $\|\cdot\|$ is equivalent to the usual Sobolev norm $\|\cdot\|_{1,2}$.

From now on we take $\left(H^{1}(\Omega),\langle\cdot\rangle,\|\cdot\|\right)$ as our working space, $\theta>\max \left\{-\lambda_{1}, 0\right\},\left\|\phi_{1}\right\|=1$. In view of Lemma 1 and the Rellich-Kondrachov Compactness theorem (see [15, Theorem 1]), we directly get the following lemma.

Lemma 2. Let $\beta \in L^{s}(\Omega)$ with $s>N / 2$ and it may change sign. Then the space $H^{1}(\Omega)$ is compactly embedded in $L^{p}(\Omega)$ for $1 \leq p<2^{*}$ and continuously embedded in $L^{2^{*}}(\Omega)$; hence there exists $S>0$ such that

$$
\|u\|_{p} \leq S\|u\|, \quad \forall u \in H^{1}(\Omega) .
$$

In addition, we also need the following lemmas.

Lemma 3 (from Lemma 4.6 of [13]). Let $X$ be a Hilbert space with orthonormal direct sum splitting $X=V \oplus Z \oplus W$. Moreover, let $\operatorname{dim}(V \oplus Z)<\infty$. For $\rho>R>0$, set

$$
\begin{gathered}
A=\{u \in W:\|u\| \geq R\} \cup\{u \in Z \oplus W:\|u\|=R\}, \\
B=\{u \in V \oplus Z:\|u\|=\rho\} .
\end{gathered}
$$

Then $A$ and B link.

Lemma 4 (from Theorem 8.1 of [16]). Let $X=X_{1} \oplus X_{2}$ be a Hilbert space where $X_{1}$ has finite dimension and $J \in C^{1}(X, R)$ satisfying the (P.S.) condition and such that, for given $\rho_{1}, \rho_{2}>$ 0 ,

$$
\sup _{u \in \rho_{1} S_{1}} J(u)<a=\inf _{u \in \rho_{2} B_{2}} J(u) \leq b=\sup _{u \in \rho_{1} B_{1}} J(u)<\inf _{u \in \rho_{2} S_{2}} J(u),
$$

where $B_{i}$ and $S_{i}$ represent the unit ball and the unit sphere in $X_{i}: i=1,2$. Then there exists a critical point $u_{0}$ such that $J\left(u_{0}\right) \in[a, b]$.

Next, let $F(x, t)=\int_{0}^{t} f(x, s) d s$; in order to state our main results, we introduce the following assumptions on the nonlinear term:

$\left(f_{1}\right) \lim _{|t| \rightarrow \infty}(f(x, t) t /|t|)=+\infty$ uniformly with respect to $x \in \Omega$;

$\left(f_{2}\right) \lim _{|t| \rightarrow \infty} F(x, t)=+\infty$ uniformly with respect to $x \in$ $\Omega$;

$\left(f_{3}\right) \lim _{|t| \rightarrow \infty}(f(x, t) t /|t|)=-\infty$ uniformly with respect to $x \in \Omega$;

$\left(f_{4}\right) \lim _{|t| \rightarrow \infty} F(x, t)=-\infty$ uniformly with respect to $x \epsilon$ $\Omega$.

Our main results are given by the following theorems.

Theorem 5. Let $\lambda_{k}(k \geq 2)$ be an eigenvalue of multiplicity $m$ and $\beta \in L^{s}(\Omega)$ with $s>N / 2$ and it may change sign. Suppose that the conditions $\left(f_{0}\right)$ and $\left(f_{\infty}\right)$ hold and one of the sets of hypotheses $\left(f_{1}\right)$ and $\left(f_{2}\right)$. Then there exists $\delta_{0}>0$ such that for $\lambda \in\left(\lambda_{k}-\delta_{0}, \lambda_{k}\right)$ problem (1) has at least two solutions.

Theorem 6. Let $\lambda_{k}(k \geq 2)$ be an eigenvalue of multiplicity $m$ and $\beta \in L^{s}(\Omega)$ with $s>N / 2$ and it may change sign. Suppose that the conditions $\left(f_{0}\right)$ and $\left(f_{\infty}\right)$ hold and one of the sets of hypotheses $\left(f_{3}\right)$ and $\left(f_{4}\right)$. Then there exists $\delta_{1}>0$ such that for $\lambda \in\left(\lambda_{k}, \lambda_{k}+\delta_{1}\right)$ problem (1) has at least two solutions.

Theorem 7. Let $\beta \in L^{s}(\Omega)$ with $s>N / 2$ and it may change sign. Suppose that the conditions $\left(f_{0}\right)$ and $\left(f_{\infty}\right)$ hold, and the nonlinearity $f$ satisfies $\left(f_{2}\right)$. Then, for $\lambda<\lambda_{1}$ sufficiently close to $\lambda_{1}$, problem (1) has at least three solutions.

\section{Proof of Theorems}

The associated functional of problem (1) is

$$
\begin{aligned}
J(u)= & \frac{1}{2} \int_{\Omega}\left(|\nabla u|^{2}+\beta(x) u^{2}\right) d x-\frac{\lambda}{2} \int_{\Omega} u^{2} d x \\
& -\int_{\Omega} F(x, u) d x \\
= & \frac{1}{2}\|u\|^{2}-\frac{\lambda+\theta}{2} \int_{\Omega} u^{2} d x-\int_{\Omega} F(x, u) d x
\end{aligned}
$$


for $u \in H^{1}(\Omega)$. Under the conditions $\left(f_{0}\right)$ and $\left(f_{\infty}\right)$, it is easy to verify that, for every $\lambda \in R, J \in C^{1}\left(H^{1}(\Omega), R\right)$ and

$$
\begin{aligned}
\left\langle J^{\prime}(u), v\right\rangle= & \int_{\Omega}(\nabla u \nabla v+\beta(x) u v) d x-\lambda \int_{\Omega} u v d x \\
& -\int_{\Omega} f(x, u) v d x
\end{aligned}
$$

for $u, v \in H^{1}(\Omega)$. Moreover, critical points of $J$ are exactly weak solutions of problem (1).

It follows from $\left(f_{0}\right)$ and $\left(f_{\infty}\right)$ that for every $\varepsilon>0$ there exist $\widetilde{M}_{\varepsilon}>0$ and $L_{\widetilde{M}_{\varepsilon}} \in L^{2}(\Omega)$ such that

$$
|f(x, t)| \leq \varepsilon|t|+\left|L_{\widetilde{M}_{\varepsilon}}(x)\right|,
$$

for all $t \in R$ and a.e. $x \in \Omega$, which implies that

$$
|F(x, t)| \leq \frac{\varepsilon}{2}|t|^{2}+\left|L_{\widetilde{M}_{\varepsilon}}(x)\right||t|,
$$

for all $t \in R$ and a.e. $x \in \Omega$. From this and Hölder's inequality, we have

$$
\left|\int_{\Omega} F(x, u) d x\right| \leq \frac{\varepsilon}{2} S^{2}\|u\|^{2}+\widetilde{L}_{\varepsilon}\|u\|,
$$

where $\widetilde{L}_{\varepsilon}=S\left\|L_{\widetilde{M}_{\varepsilon}}\right\|_{2}$ and $S$ is the best embedding constant.

In addition, we will use several times the estimates below.

From (7) and (8), we have

$$
\|u\|^{2} \geq\left(\theta+\lambda_{1}\right) \int_{\Omega} u^{2} d x, \quad \forall u \in H^{1}(\Omega),
$$

$$
\|u\|^{2} \leq\left(\theta+\lambda_{k}\right) \int_{\Omega} u^{2} d x, \quad \forall u \in \operatorname{span}\left\{\phi_{1}, \phi_{2}, \ldots, \phi_{k}\right\},
$$

$\|u\|^{2} \geq\left(\theta+\lambda_{k+1}\right) \int_{\Omega} u^{2} d x, \quad \forall u \in\left(\operatorname{span}\left\{\phi_{1}, \phi_{2}, \ldots, \phi_{k}\right\}\right)^{\perp}$.

Proposition 8. Assume that $\left(f_{0}\right)$ and $\left(f_{\infty}\right)$ hold, and suppose that $\lambda \neq \lambda_{k}$ for any $k \in N^{+}$. Then J satisfies the (P.S.) condition.

Proof. For any sequence $\left\{u_{n}\right\} \subset H^{1}(\Omega)$ such that

$$
\left|J\left(u_{n}\right)\right|<\infty \quad \forall n, \quad J^{\prime}\left(u_{n}\right) \longrightarrow 0 \quad \text { as } n \longrightarrow \infty,
$$

we need to prove that $\left\{u_{n}\right\}$ has a convergent subsequence. By the standard argument, it suffices to show that $\left\{u_{n}\right\}$ is bounded in $H^{1}(\Omega)$. Suppose by contradiction that $\left\|u_{n}\right\|$ as $n \longrightarrow \infty$. Let $w_{n}=u_{n} /\left\|u_{n}\right\|$. Then $\left\|w_{n}\right\|=1$, so we may suppose that there is $w \in H^{1}(\Omega)$ such that

$$
w_{n} \rightarrow w \text { weakly in } H^{1}(\Omega),
$$

$w_{n} \longrightarrow w$ strongly in $L^{p}(\Omega)$, where $p \in\left[1,2^{*}\right)$, as $n \longrightarrow \infty$. Then, for any $v \in H^{1}(\Omega)$, from (24) and (27), we have

$$
\begin{aligned}
\frac{1}{\left\|u_{n}\right\|} \int_{\Omega} f\left(x, u_{n}\right) v d x & \leq \frac{1}{\left\|u_{n}\right\|} \int_{\Omega}\left(\varepsilon\left|u_{n}\right|+\left|L_{\widetilde{M}_{\varepsilon}}(x)\right|\right)|v| d x \\
& \leq \frac{1}{\left\|u_{n}\right\|}\left(\varepsilon\left\|u_{n}\right\|_{2}\|v\|_{2}+\left\|L_{\widetilde{M}_{\varepsilon}}\right\|\left\|_{2}\right\| v \|_{2}\right) \\
& \leq \frac{\varepsilon}{\sqrt{\theta+\lambda_{1}}}\|v\|_{2}+\frac{\left\|L_{\widetilde{M}_{\varepsilon}}\right\|\left\|_{2}\right\| v \|_{2}}{\left\|u_{n}\right\|}
\end{aligned}
$$

which shows that

$$
\lim _{n \rightarrow \infty} \frac{1}{\left\|u_{n}\right\|} \int_{\Omega} f\left(x, u_{n}\right) v d x \leq \frac{\varepsilon}{\sqrt{\theta+\lambda_{1}}}\|v\|_{2} .
$$

By the arbitrariness of $\varepsilon$, one has

$$
\lim _{n \longrightarrow \infty} \frac{1}{\left\|u_{n}\right\|} \int_{\Omega} f\left(x, u_{n}\right) v d x=0 .
$$

Like in the proof of (34), from (26), it follows that

$$
\lim _{n \rightarrow \infty} \frac{1}{\left\|u_{n}\right\|^{2}} \int_{\Omega} F\left(x, u_{n}\right) v d x=0 .
$$

Thus, for any $v \in H^{1}(\Omega)$, by (30), (31), and (34), passing to the limit in

$$
\begin{aligned}
\frac{\left\langle J^{\prime}\left(u_{n}\right), v\right\rangle}{\left\|u_{n}\right\|}= & \int_{\Omega}\left(\nabla w_{n} \nabla v+\beta(x) w_{n} v\right) d x \\
& -\lambda \int_{\Omega} w_{n} v d x-\int_{\Omega} f\left(x, u_{n}\right) v d x
\end{aligned}
$$

gives

$$
\int_{\Omega}\left(\nabla w_{n} \nabla v d x+\beta(x) w_{n} v\right) d x-\lambda \int_{\Omega} w_{n} v d x=0,
$$

which implies that

$$
w \in \operatorname{Ker}(-\Delta+\beta-\lambda) .
$$

In addition, by (30), (31), and (35), passing to the limit in

$$
\begin{aligned}
\frac{J\left(u_{n}\right)}{\left\|u_{n}\right\|^{2}}= & \frac{1}{2} \int_{\Omega}\left(\left|\nabla w_{n}\right|^{2}+\beta(x) w_{n}^{2}+\theta w_{n}^{2}\right) d x \\
& -\frac{\theta+\lambda}{2} \int_{\Omega} w_{n}^{2} d x-\frac{1}{\left\|u_{n}\right\|^{2}} \int_{\Omega} F\left(x, u_{n}\right) d x
\end{aligned}
$$

gives

$$
\frac{1}{2}-\frac{\theta+\lambda}{2} \int_{\Omega} w^{2} d x=0
$$

If $\theta+\lambda=0$, then, from (40), we have $1 / 2=0$, which is a contradiction.

If $\theta+\lambda \neq 0$, then, from (40), we have $w \neq 0$. From this and (38) it follows that $\lambda$ is an eigenvalue of operator $-\Delta+\beta$, a contradiction; the proof is completed. 
Hereafter, we set

$$
\begin{gathered}
V=\operatorname{span}\left\{\phi_{1}, \ldots, \phi_{k-1}\right\}, \\
Z=\operatorname{span}\left\{\phi_{k}, \ldots, \phi_{k+m-1}\right\}=E_{k}, \\
W=(V \oplus Z)^{\perp},
\end{gathered}
$$

and we define

$$
\begin{gathered}
B_{V}=\{u \in V:\|u\| \leq 1\}, \quad B_{V Z}=\{u \in V \oplus Z:\|u\| \leq 1\}, \\
B_{Z W}=\{u \in Z \oplus W:\|u\| \leq 1\},
\end{gathered}
$$

and $S_{V}, S_{V Z}$, and $S_{Z W}$, respectively, are their relative boundaries.

Theorems 5 and 6 will be a consequence of the geometry in Propositions 9 and 10 stated below, whose proofs will be postponed to Sections 4 and 5.

Proposition 9. If $\lambda \in\left(\lambda_{k-1}, \lambda_{k}\right)$ and hypotheses $\left(f_{0}\right)$ and $\left(f_{\infty}\right)$ are satisfied, then there exist constants $D_{\lambda}$ and $\rho_{\lambda}$ such that

$$
\begin{gathered}
J(u) \geq D_{\lambda}, \quad \text { for } u \in Z \oplus W, \\
J(u)<D_{\lambda}, \quad \text { for } u \in \rho_{\lambda} S_{V} .
\end{gathered}
$$

Moreover, if one of the sets of hypotheses $\left(f_{1}\right)$ and $\left(f_{2}\right)$ is satisfied, then there exists $\delta_{0}$ such that for $\lambda \in\left(\lambda_{k}-\delta_{0}, \lambda_{k}\right)$ there exist $D_{W}, D_{\lambda} \in R, \rho_{\lambda}>R_{1}>0$ such that, in addition to (43) and (44),

$$
\begin{gathered}
J(u) \geq D_{W}, \quad \text { for } u \in W, \\
J(u)<D_{W}, \quad \text { for } u \in R_{1} S_{V Z}, \\
J(u)<D_{W}, \quad \text { for } u \in V,\|z\| \geq R_{1} .
\end{gathered}
$$

(The values with index $\lambda$ depend on $\lambda$; the others may be fixed uniformly.)

Proposition 10. If $\lambda \in\left(\lambda_{k}, \lambda_{k+m}\right)$ and hypotheses $\left(f_{0}\right)$ and $\left(f_{\infty}\right)$ are satisfied, then there exist constants $K_{\lambda}$ and $\beta_{\lambda}$ such that

$$
\begin{gathered}
J(z) \geq K_{\lambda}, \quad \text { for } z \in W, \\
J(z)<K_{\lambda}, \quad \text { for } z \in \beta_{\lambda} S_{V Z} .
\end{gathered}
$$

Moreover, if one of the sets of hypotheses $\left(f_{3}\right)$ and $\left(f_{4}\right)$ is satisfied, then there exists $\delta_{1}$ such that for $\lambda \in\left(\lambda_{k}, \lambda_{k}+\delta_{1}\right)$ there exist $K_{\lambda}, K_{V}, E \in R, \beta_{\lambda}>R_{2}>0$, and $\xi>0$ such that, in addition to (48) and (49),

$$
\begin{gathered}
J(u)<K_{V}, \quad \text { for } u \in V, \\
J(u)>K_{V}, \quad \text { for } u \in R_{2} S_{Z W}, \\
J(u)>K_{V}, \quad \text { for } u \in W,\|u\| \geq R_{2}, \\
J(u)>E, \quad \text { for } u \in R_{2} B_{Z W}, \\
J(u)<E, \quad \text { for } u \in \xi S_{V} .
\end{gathered}
$$

(The values with index $\lambda$ depend on $\lambda$; the others may be fixed uniformly.)
Proof of Theorem 5. Since the functional $J$ satisfies the (P.S.) condition, we can apply two times the saddle point theorem (see, e.g., [17]); let

$$
\begin{aligned}
& \Gamma_{k-1}=\left\{\gamma \in C^{0}\left(\rho_{\lambda} B_{V} ; E\right) \text { s.t. }\left.\gamma\right|_{\rho_{\lambda S_{V}}}=\mathrm{id}\right\}, \\
& \Gamma_{k}=\left\{\gamma \in C^{0}\left(R_{1} B_{V Z} ; E\right) \text { s.t. }\left.\gamma\right|_{R_{1} S_{V Z}}=\mathrm{id}\right\} .
\end{aligned}
$$

The first solution, which we denote by $u_{k-1}$ and may be obtained for any $\lambda \in\left(\lambda_{k-1}, \lambda_{k}\right)$ with just hypotheses $\left(f_{0}\right)$ and $\left(f_{\infty}\right)$, corresponds to a critical point at the level

$$
c_{k-1}=\inf _{\gamma \in \Gamma_{k-1}} \sup _{w \in \rho_{\lambda B_{V}}} J(\gamma(w)) \text {. }
$$

The criticality of this level is guaranteed by the estimates (43) and (44), since $\rho_{\lambda} S_{V}$ and $Z \oplus W$ link; that is, the image of any map in $\Gamma_{k-1}$ intersects $Z \oplus W$.

The second solution, which we denote by $u_{k}$, corresponds to a critical point at the critical level

$$
c_{k}=\inf _{\gamma \in \Gamma_{k}} \sup _{w \in R_{1} B_{V Z}} J(\gamma(w)) .
$$

Actually, this is a critical level because of the estimates (45) and (46), since $R_{1} S_{V Z}$ and $W$ link.

To conclude the proof, we need to show that these two solutions are distinct.

We observe first that by estimate (45) we have that $c_{k} \geq$ $D_{W}$, then we observe that we may build a map $\gamma_{0} \in \Gamma_{k-1}$ in such a way that its image is the union between the annulus $\left\{u \in V:\|u\| \in\left[R_{1}, \rho_{\lambda}\right]\right\}$ and the image of a $(k-1)$-dimensional ball in $R_{1} S_{V Z}$ whose boundary is $R_{1} S_{V}$. By the estimates (46) and (47), we deduce that $\sup _{w \in \rho_{\lambda} B_{V}} J\left(\gamma_{0}(w)\right)<D_{W}$, and as a consequence $c_{k-1}<D_{W}$, proving that the two solutions are distinct, for being at different critical levels.

Proof of Theorem 6. Since the functional $J$ satisfies the (P.S.) condition, we can apply the saddle point theorem and Lemma 4.

The first solution, which we denote by $w_{k}$ and may be obtained for any $\lambda \in\left(\lambda_{k}, \lambda_{k+m}\right)$ with just hypotheses $\left(f_{0}\right)$ and $\left(f_{\infty}\right)$, is again obtained through the saddle point theorem and corresponds to a critical point at the critical level

$$
d_{k}=\inf _{\gamma \in \Gamma_{k}} \sup _{w \in \beta_{\lambda} B_{V Z}} J(\gamma(w))
$$

where now

$$
\Gamma_{k}=\left\{\gamma \in C^{0}\left(\beta_{\lambda} B_{V Z} ; E\right) \text { s.t. }\left.\gamma\right|_{\beta_{\lambda} S_{V Z}}=\mathrm{id}\right\} .
$$

The criticality is guaranteed by estimates (48) and (49), since $\beta_{\lambda} S_{V Z}$ and $W$ link.

The second solution, which we denote by $w_{k-1}$, comes from Lemma 3, where we set $X_{1}=V$ and $X_{2}=Z \oplus W$; actually we have the structure

$$
\sup _{\xi S_{V}} J(u)<E=\inf _{R_{2} B_{Z W}} J(u) \leq \sup _{\xi B_{V}} J(u)<K_{V}<\inf _{R_{2} S_{Z W}} J(u)
$$

and then we have a critical point $w_{k-1}$ at the level $d_{k-1} \leq K_{V}$. 
Finally, in order to prove that these two solutions are distinct, we need a sharper estimate for $d_{k}$ than that given by (49). For this we use Lemma 3 to guarantee that, for any map $\gamma \in \Gamma_{k}$, since $\beta_{\lambda}>R_{2}$, one has that the image of $\gamma$ either intersects $R_{2} S_{Z W}$ or has a point $u \in W$ with $\|u\| \geq R_{2}$. This implies that

$$
\sup _{w \in \beta_{\lambda} B_{V Z}} J(\gamma(w))>K_{V}
$$

by estimates (51) and (52), and then $d_{k}>K_{V}$ proving that the two solutions are distinct, for being at different critical levels.

Proof of Theorem 7. The proof will be divided into four steps.

Step 1. For $\lambda<\lambda_{1}$, from the definition of $\lambda_{1}$, (26), and (27), we get

$$
J(u) \geq \frac{1}{2}\left(\frac{\lambda_{1}-\lambda}{\theta+\lambda_{1}}-\varepsilon S^{2}\right)\|u\|^{2}-\widetilde{L}_{\varepsilon}\|u\|
$$

Letting $\varepsilon=\left(\lambda_{1}-\lambda\right) / 2 S^{2}\left(\theta+\lambda_{1}\right)$, it follows that $J$ is coercive in $H^{1}(\Omega)$.

Similarly, from (26) and (29), we obtain

$$
\begin{aligned}
J(w) & \geq \frac{1}{2}\left(\frac{\lambda_{2}-\lambda}{\theta+\lambda_{2}}-\varepsilon S^{2}\right)\|w\|^{2}-\widetilde{L}_{\varepsilon}\|w\| \\
& \geq \frac{1}{2}\left(\frac{\lambda_{2}-\lambda_{1}}{\theta+\lambda_{2}}-\varepsilon S^{2}\right)\|w\|^{2}-\widetilde{L}_{\varepsilon}\|w\|,
\end{aligned}
$$

for all $w \in\left(\operatorname{span}\left\{\phi_{1}\right\}\right)^{\perp}$. Let $\varepsilon=\left(\lambda_{2}-\lambda_{1}\right) / 2 S^{2}\left(\theta+\lambda_{2}\right)$; hence $J$ is coercive in $\left(\operatorname{span}\left\{\phi_{1}\right\}\right)^{\perp}$ and $J$ is bounded from below on $\left(\operatorname{span}\left\{\phi_{1}\right\}\right)^{\perp}$, and, moreover, there is a constant $M$, independent of $\lambda$, such that $\inf _{\left(\operatorname{span}\left\{\phi_{1}\right\}\right)^{\perp}} J \geq M$.

Step 2. If $\lambda<\lambda_{1}$ is sufficiently close to $\lambda_{1}$, we have $t^{-}<0<t^{+}$ such that $J\left(t^{ \pm} \phi_{1}\right)<M$. In fact, for $\lambda<\lambda_{1}$, we have

$$
J\left(t \phi_{1}\right)=\frac{\lambda_{1}-\lambda}{2\left(\theta+\lambda_{1}\right)}\left\|t \phi_{1}\right\|^{2}-\int_{\Omega} F\left(x, t \phi_{1}\right) d x .
$$

For any fixed $\widetilde{u} \in R$ with $|\widetilde{u}|=1$, from $\left(f_{2}\right)$, we get $\lim _{|t| \rightarrow \infty} F(x, t \tilde{u})=+\infty$ uniformly in $x \in \Omega$. From Fatou's lemma and $\phi_{1}>0$ in $\Omega$, it follows that

$$
\lim _{t \rightarrow+\infty} \int_{\Omega} F\left(x, t \phi_{1}\right) d x \geq \int_{\Omega} \lim _{t \rightarrow+\infty} F\left(x, t \phi_{1}\right) d x=+\infty,
$$

and hence, taking $t^{+}(>0)$ large enough, we get

$$
\int_{\Omega} F\left(x, t^{+} \phi_{1}\right) d x>-M+1
$$

For $\lambda_{1}-2\left(\theta+\lambda_{1}\right) /\left(t^{+}\right)^{2}<\lambda<\lambda_{1}$, combining (64) and (66) yields $J\left(t^{+} \phi_{1}\right)<M$. A similar conclusion holds for some $t^{-}<$ 0 .

Step 3. If $\lambda<\lambda_{1}$, let

$$
\begin{gathered}
\Sigma_{ \pm}=\left\{z \in H^{1}(\Omega): z= \pm t \phi_{1}+w \text { with } t>0,\right. \\
\left.w \in\left(\operatorname{span}\left\{\phi_{1}\right\}\right)^{\perp}\right\} .
\end{gathered}
$$

The functional $J$ satisfies the (P.S. $)_{c, \Sigma_{+}}$and (P.S. $)_{c, \Sigma_{-}}$condition for all $c<M$.

In fact, let $\left\{z_{n}\right\} \subset \sum_{+}$satisfy $J\left(z_{n}\right) \longrightarrow c<M$ and $J^{\prime}\left(z_{n}\right) \longrightarrow 0$ as $n \longrightarrow \infty$. From Proposition 8 , there is $z \in H^{1}(\Omega)$ such that $z_{n} \longrightarrow z$ strongly in $H^{1}(\Omega)$. If $z \in$ $\partial \Sigma_{+}=\left(\operatorname{span}\left\{\phi_{1}\right\}\right)^{\perp}$, from the second conclusion of Step 1, we get $J\left(z_{n}\right) \longrightarrow c \geq M$, which is impossible. Hence, $c \in \Sigma_{+}$and $J$ satisfies the (P.S.) $)_{c, \Sigma_{+}}$. Similarly we have that (P.S.) $)_{c, \Sigma_{-}}$holds for all $c<M$.

Step 4. Three solutions are obtained.

If $\lambda<\lambda_{1}$ is sufficiently close to $\lambda_{1}$, from Steps 1 and 2, we get $-\infty<\inf _{\Sigma_{+}} J<M$, which implies that $J$ is bounded below in $\Sigma_{+}$. Consequently, from Ekeland's variational principle, there exists $\left\{z_{n}\right\} \subset \Sigma_{+}$such that $J\left(z_{n}\right) \longrightarrow \inf _{\Sigma_{+}} J$ and $J^{\prime}\left(z_{n}\right) \longrightarrow 0$ as $n \longrightarrow \infty$. Since $J$ satisfies (P.S.) $)_{c, \Sigma_{+}}$for all $c<M$, there is $z^{+} \in \Sigma_{+}$such that $J\left(z^{+}\right)=\inf _{\Sigma_{+}} J$; that is, the infimum is attained in $\Sigma_{+}$. A similar conclusion holds in $\Sigma_{-}$. So $J$ has two distinct critical points, denoted by $z^{+}, z^{-}$.

Suppose that $J\left(z^{+}\right) \geq J\left(z^{-}\right)$. Letting

$$
\psi(z)=J\left(z+z^{+}\right)-J\left(z^{+}\right), \quad e=z^{-}-z^{+},
$$

we have $\psi(0)=0, \psi(e) \leq 0$ and since $z^{+}$is the local minimum of $J$, there are $r, \rho>0$ such that $\psi(z) \geq \rho$ for $\|z\|=r$. Since $\psi^{\prime}=J^{\prime}$ and $\psi$ satisfies the (P.S.) condition, from the mountain pass theorem, the number

$$
c=\inf _{h \in \Gamma} \max _{t \in[0,1]} J(h(t)),
$$

where $\Gamma=\left\{h \in C\left([0,1], H^{1}(\Omega)\right): h(0)=z^{+}, h(1)=z^{-}\right\}$, is a critical value of $J$. From the definition of $c$, we have $c \geq$ $M$ and obtain a third critical point of $J$. Hence, the proof is completed.

\section{Proof of Estimates}

In this section we will prove all the estimates in Propositions 9 and 10 .

\subsection{Estimates of the Saddle Geometry}

Lemma 11. Under hypotheses $\left(f_{0}\right)$ and $\left(f_{\infty}\right)$, one gets the following:

(i) for $\lambda \in\left(\lambda_{k-1}, \lambda_{k}\right)$, there exists $D_{\lambda}$ satisfying (43) and $D_{W} \in R$ satisfying (45);

(ii) for $\lambda \in\left(\lambda_{k}, \lambda_{k+m}\right)$ one has the following:

(a) there exists $K_{\lambda} \in R$ satisfying (48),

(b) for a given $R_{2}>0$, there exists $E \in R$ satisfying (53).

Proof. Let $u \in W$; using estimates (26) and (29) we get

$$
J(u) \geq \frac{1}{2}\left(\frac{\lambda_{k+m}-\lambda}{\theta+\lambda_{k+m}}-\varepsilon S^{2}\right)\|u\|^{2}-\widetilde{L}_{\varepsilon}\|u\| .
$$


For $\lambda \in\left(\lambda_{k-1}, \lambda_{k}\right)$, letting $\varepsilon<\left(\lambda_{k+m}-\lambda_{k}\right) / S^{2}\left(\theta+\lambda_{k+m}\right)<$ $\left(\lambda_{k+m}-\lambda\right) / S^{2}\left(\theta+\lambda_{k+m}\right)$, it follows that $J$ is bounded below in $W$; that is, there exists a $D_{W}$ as in (45).

For $\lambda \in\left(\lambda_{k}, \lambda_{k+m}\right)$, then the same estimate holds but the constant cannot be made independent of $\lambda$, giving (48).

In the same way, let $u \in Z \oplus W$ and set $\delta=\lambda_{k}-\lambda>0$; we get

$$
\begin{aligned}
J(u) & \geq \frac{1}{2}\left(\frac{\lambda_{k}-\lambda}{\theta+\lambda_{k}}-\varepsilon S^{2}\right)\|u\|^{2}-\widetilde{L}_{\varepsilon}\|u\| \\
& \geq \frac{1}{2}\left(\frac{\delta}{\theta+\lambda_{k}}-\varepsilon S^{2}\right)\|u\|^{2}-\widetilde{L}_{\varepsilon}\|u\| .
\end{aligned}
$$

Letting $\varepsilon<\delta / S^{2}\left(\theta+\lambda_{k}\right)$, it follows that $J$ is bounded below in $Z \oplus W$; that is, there exists a $D_{\lambda}$ such that for all $u \in Z \oplus W$ we have (43), where again the constant $D_{\lambda}$ depends on $\delta$, that is, on $\lambda$.

Finally, (71) with $\lambda \in\left(\lambda_{k}, \lambda_{k+m}\right)$ implies

$$
J(u) \geq \frac{1}{2}\left(\frac{\lambda_{k}-\lambda_{k+m}}{\theta+\lambda_{k}}-\varepsilon S^{2}\right)\|u\|^{2}-\widetilde{L}_{\varepsilon}\|u\| .
$$

Then, no matter the value of $\lambda, J$ is bounded from below in any bounded subset of $Z \oplus W$, giving (53) for a suitable value of $E$.

Lemma 12. Under hypotheses $\left(f_{0}\right)$ and $\left(f_{\infty}\right)$, one gets the following:

(i) for $\lambda \in\left(\lambda_{k-1}, \lambda_{k}\right)$, given the constant $D_{\lambda} \in R$, there exists $\rho_{\lambda}>0$ satisfying (44);

(ii) for $\lambda \in\left(\lambda_{k}, \lambda_{k+m}\right)$ one has the following:

(a) there exists $K_{V} \in R$ satisfying (50),

(b) for a given $K_{\lambda} \in R$, there exists $\beta_{\lambda}>0$ satisfying (49),

(c) for a given $E \in R$, there exists $\xi>0$ satisfying (54).

Moreover, given the values $R_{1}, R_{2}$, one may always choose $\rho_{\lambda}>R_{1}, \beta_{\lambda}>R_{2}$ as claimed in Propositions 9 and 10.

Proof. Let $u \in V$, by estimates (26) and (28); we get

$$
J(u) \leq \frac{1}{2}\left(\frac{\lambda_{k-1}-\lambda}{\theta+\lambda_{k-1}}+\varepsilon S^{2}\right)\|u\|^{2}+\widetilde{L}_{\varepsilon}\|u\| .
$$

For $\lambda \in\left(\lambda_{k-1}, \lambda_{k}\right)$, letting $\varepsilon<\left(\lambda-\lambda_{k-1}\right) / S^{2}\left(\theta+\lambda_{k-1}\right)$, then one obtains (44) for suitably large $\rho_{\lambda}>R_{1}$.

For $\lambda \in\left(\lambda_{k}, \lambda_{k+m}\right)$, letting $\varepsilon<\left(\lambda_{k}-\lambda_{k-1}\right) / S^{2}\left(\theta+\lambda_{k-1}\right)$, one obtains, for suitable $K_{V}$ and $\xi>0$, (50) and (54).

Finally, letting $u \in V \oplus Z$ and setting $\delta=\lambda-\lambda_{k}>0$, we get

$$
\begin{aligned}
J(u) & \leq \frac{1}{2}\left(\frac{\lambda_{k}-\lambda}{\theta+\lambda_{k}}+\varepsilon S^{2}\right)\|u\|^{2}+\widetilde{L}_{\varepsilon}\|u\| \\
& \leq \frac{1}{2}\left(-\frac{\delta}{\theta+\lambda_{k}}+\varepsilon S^{2}\right)\|u\|^{2}+\widetilde{L}_{\varepsilon}\|u\| .
\end{aligned}
$$

Letting $\varepsilon<\delta / S^{2} \lambda_{k}$, it is clear that (once $\delta$ is fixed) this goes to $-\infty$ and then we may find the claimed $\beta_{\lambda}>R_{2}$ such that (49) holds.

Observe that $K_{V}$ and $E$ can be chosen uniformly for $\lambda \epsilon$ $\left(\lambda_{k}, \lambda_{k+m}\right)$, while $\rho_{\lambda}, \beta_{\lambda}$ will in fact depend on $\lambda$.

4.2. Estimating the Effect of the Nontrivial Perturbation. In this section we will prove the remaining inequalities in Propositions 9 and 10, those which rely on the hypothesis $\left(f_{1}\right)$ or $\left(f_{2}\right)$ or $\left(f_{3}\right)$ or $\left(f_{4}\right)$, which, roughly speaking, say that the perturbation $F$ is nontrivial in such a way that a new solution arises when $\lambda$ is sufficiently near to the eigenvalue $\lambda_{k}$. The proof is simpler for Theorem 5 , since we need to estimate the functional in the compact set $S_{V Z}$, while for Theorem 6 the same kind of estimate is required in the noncompact set $S_{Z W}$.

4.2.1. Estimating $J$ in $S_{V Z}$. For the next estimates, we will need the following lemma.

Lemma 13. Hypothesis $\left(f_{2}\right)$ implies that there exists a nondecreasing function $D:(0,+\infty) \longrightarrow R$ such that

$$
\lim _{R \longrightarrow+\infty} D(R)=+\infty, \quad \inf _{u \in R S_{V Z}} \int_{\Omega} F(x, u) d x>D(R) .
$$

Proof. First we claim that there exists a constant $\eta>0$ such that the sets $\Omega_{u}=\{x \in \Omega:|u(x)|>\eta\}$ have measure $\left|\Omega_{u}\right|>$ $\eta$, for all $u \in S_{V Z}$.

Actually, $V \oplus Z$ is a finite-dimensional subspace and the functions $u \in S_{V Z}$ are smooth; they are uniformly bounded; that is, there exists $M>0$ such that $|u(x)| \leq M$ for all $x \in \Omega$. Suppose that for $\eta_{n} \longrightarrow 0\left(\eta_{n}<1\right)$ there exists $\left\{u_{n}\right\} \subset S_{V Z}$ such that $\left|\Omega_{u_{n}}\right| \leq \eta_{n}$.

On one hand, by (28), one obtains

$$
\frac{1}{\theta+\lambda_{k}} \leq \int_{\Omega} u^{2} d x
$$

On the other hand,

$$
\begin{aligned}
\int_{\Omega} u^{2} d x & =\int_{\Omega_{u_{n}}}\left|u_{n}\right|^{2} d x+\int_{\Omega-\Omega_{u_{n}}}\left|u_{n}\right|^{2} d x \\
& \leq M^{2}\left|\Omega_{u_{n}}\right|+\eta_{n}^{2}\left|\Omega-\Omega_{u_{n}}\right| \\
& \leq \eta_{n}\left(M^{2}+|\Omega|\right) \\
& \longrightarrow 0 .
\end{aligned}
$$

That is a contradiction.

Now, for any $H>0$, we will show that we can find a $\widetilde{R}$ large enough so that $\int_{\Omega} F(x, R u) d x \geq H$ for any $u \in S_{V Z}$ and $R \geq \widetilde{R}$; this means that

$$
\lim _{R \longrightarrow \infty} \inf _{u \in R S_{V Z}} \int_{\Omega} F(x, u) d x=+\infty .
$$


Actually, it follows from $\left(f_{2}\right)$ that for any $M>0$ there exists $t_{0}(>0)$ such that $F(x, t)>M$ for $|t|>t_{0}$. For $R>t_{0} / \eta$, one has $\Omega_{u} \subseteq\left\{x \in \Omega:|R u(x)|>t_{0}\right\}$, and then one gets

$$
\int_{|R u| \geq t_{0}} F(x, R u) d x \geq M \eta
$$

For $R \leq t_{0} / \eta$, by $\left(f_{0}\right)$ and $\left(f_{2}\right)$, there exist $\widetilde{C}>0$ and $L_{\widetilde{C}} \in L^{2}(\Omega)$ such that $F(x, t) \geq-\widetilde{C}\left(1+L_{\widetilde{C}}(x)\right)$, for $t \in R$ and a.e. $x \in \Omega$.

Let $M=\left(H+\widetilde{C}|\Omega|+\widetilde{C}|\Omega|^{1 / 2}\left\|L_{\widetilde{C}}\right\|_{2}\right) \eta^{-1}$; one finally obtains

$$
\begin{aligned}
\int_{\Omega} F(x, R u) d x & =\int_{|R u| \geq t_{0}} F(x, R u) d x+\int_{|R u| \leq t_{0}} F(x, R u) d x \\
& \geq M \eta-\widetilde{C}|\Omega|-\widetilde{C}|\Omega|^{1 / 2} \| L_{\widetilde{C} \|_{2}} \\
& =H .
\end{aligned}
$$

It is elementary that

$$
D(R)=\inf _{\rho \geq R} \inf _{u \in R S_{V Z}} \int_{\Omega} F(x, u) d x
$$

is well defined and satisfies the claim.

Now we may prove the following.

Lemma 14. Consider Theorem 5 with one of the sets of hypotheses $\left(f_{1}\right)$ and $\left(f_{2}\right)$. Given the constant $D_{W} \in R$, there exist $R_{1}, \delta_{0}>0$ such that, for any $\lambda \in\left(\lambda_{k}-\delta_{0}, \lambda_{k}\right),(46)$ and (47) hold.

Proof. We consider the two sets of hypotheses separately.

(i) In case $\left(f_{1}\right)$, it follows from $\left(f_{0}\right)$ and $\left(f_{1}\right)$ that for any $M>0$ there exist $C_{M}$ and $L_{M} \in L^{2}(\Omega)$ such that

$$
F(x, t) \geq M|t|-C_{M}\left(1+L_{M}(x)\right)
$$

for $t \in R$ and $x \in \Omega$; in particular we set $M=1$. Let $u \in R S_{V Z}$; for being in a finite-dimensional subspace, all the norms are equivalent, so that ( $\operatorname{set} \delta=\lambda_{k}-\lambda>0$ and use estimates (28) and (82))

$$
\begin{aligned}
J(u) & \leq \frac{\lambda_{k}-\lambda}{2\left(\theta+\lambda_{k}\right)}\|u\|^{2}-\|u\|+C_{0} \\
& \leq \frac{\delta}{2\left(\theta+\lambda_{k}\right)}\|u\|^{2}-\|u\|+C_{0} \\
& \leq \frac{\delta}{2\left(\theta+\lambda_{k}\right)} R^{2}-R+C_{0},
\end{aligned}
$$

where $C_{0}=C_{1}|\Omega|^{1 / 2}\left\|L_{1}\right\|_{2}+C_{1}|\Omega|$. (ii) In case $\left(f_{2}\right)$, let $D(R)$ be as in Lemma 13 , for $\|u\|=R$; let $u=w+\phi$ with $w \in V$ and $\phi \in Z=E_{k}$ :

$$
\begin{aligned}
J(u) & \leq \frac{\lambda_{k-1}-\lambda}{2\left(\theta+\lambda_{k-1}\right)}\|w\|^{2}+\frac{\lambda_{k}-\lambda}{2\left(\theta+\lambda_{k}\right)}\|\phi\|^{2}-\int_{\Omega} F(x, u) d x \\
& \leq \frac{\lambda_{k-1}-\lambda_{k}+\delta}{2\left(\theta+\lambda_{k-1}\right)}\|w\|^{2}+\frac{\delta}{2\left(\theta+\lambda_{k}\right)}\|\phi\|^{2}-\int_{\Omega} F(x, u) d x \\
& \leq \frac{\delta}{2\left(\theta+\lambda_{k}\right)}\|\phi\|^{2}-\frac{\lambda_{k}-\lambda_{k-1}}{4\left(\theta+\lambda_{k-1}\right)}\|w\|^{2}-\int_{\Omega} F(x, u) d x .
\end{aligned}
$$

We assume that $\delta \leq\left(\lambda_{k}-\lambda_{k-1}\right) / 2$; it is easy to see that $\left(\lambda_{k-1}-\right.$ $\left.\lambda_{k}\right)\|w\|^{2} / 4\left(\theta+\lambda_{k-1}\right) \leq C$ for some constant $C$; we estimate

$$
\begin{aligned}
J(z) & \leq \frac{\delta}{2\left(\theta+\lambda_{k}\right)}\|\phi\|^{2}+C-D(R) \\
& \leq \frac{\delta}{2\left(\theta+\lambda_{k}\right)} R^{2}-D(R)+C .
\end{aligned}
$$

Considering (83) and (85), we see that since $\lim _{R \rightarrow \infty} D(R)=$ $+\infty$ by Lemma 13, we may fix $R_{1}$ so that $C-D\left(R_{1}\right)<D_{W}-1$ (or $C_{0}-R_{1}<D_{W}-1$ for the case $\left(f_{1}\right)$ ) and then for $0<\delta<$ $\min \left\{2\left(\theta+\lambda_{k}\right) / R_{1}^{2},\left(\lambda_{k}-\lambda_{k-1}\right) / 2\right\}$ one gets (46).

To obtain (47), we observe that (since $\lambda>\lambda_{k-1}$ ) if $\phi=$ 0 , that is, if $u \in V$, then in estimates (83) and (85) we may avoid the term $\delta R^{2} / 2\left(\theta+\lambda_{k}\right)$ so that (remember that $D(R)$ is nondecreasing) $J(u)<D_{W}-1$ for $\|u\|>R_{1}$.

4.2.2. Estimating $J$ in $S_{Z W}$. We consider the corresponding estimates of the previous lemma, for Theorem 6.

Lemma 15. Consider Theorem 6 with one of the sets of hypotheses $\left(f_{3}\right)$ and $\left(f_{4}\right)$. Given the constant $K_{V} \in R$, there exist $R_{2}, \delta_{1}>0$ such that, for any $\lambda \in\left(\lambda_{k}, \lambda_{k}+\delta_{1}\right)$, (51) and (52) hold.

Proof. Letting $\lambda=\lambda_{k}+\delta$, we see from (70) that property (52) will be satisfied provided that $R_{2}$ is large enough (say $R_{2}>\widetilde{R}$ ); observe that this value can be made independent from $\lambda$ once $\delta$ is small enough.

Now we consider the two sets of hypotheses separately.

(i) In case $\left(f_{3}\right)$, suppose $u \in E_{k} \oplus W$; we can assume that $u=w+\phi$, with $w \in W$ and $\phi \in E_{k}$. Since $E_{k}$ is a finite dimension subspace, all the norms are equivalent, so that there exists $K>0$ such that for all $\phi \in E_{k}$ we have $\|\phi\| \leq K\|\phi\|_{1}$. In addition, by $\left(f_{0}\right)$ and $\left(f_{3}\right)$, there exist $C_{2}$ and $L_{2} \in L^{2}(\Omega)$ such that

$$
-F(x, s) \geq K|s|-C_{2}\left(1+L_{2}(x)\right),
$$


uniformly in $x \in \Omega$. So by (29) and (86),

$$
\begin{aligned}
J(w+\phi) \geq & \frac{\lambda_{k+m}-\left(\lambda_{k}+\delta\right)}{2\left(\theta+\lambda_{k+m}\right)}\|w\|^{2}-\frac{\delta}{2\left(\theta+\lambda_{k}\right)}\|\phi\|^{2} \\
& +K\|w+\phi\|_{1}-C_{4} \\
\geq & \frac{\lambda_{k+m}-\left(\lambda_{k}+\delta\right)}{2\left(\theta+\lambda_{k+m}\right)}\|w\|^{2}-\frac{\delta}{2\left(\theta+\lambda_{k}\right)}\|\phi\|^{2} \\
& +K\|\phi\|_{1}-K\|-w\|_{1}-C_{4} \\
\geq & \frac{\lambda_{k+m}-\left(\lambda_{k}+\delta\right)}{2\left(\theta+\lambda_{k+m}\right)}\|w\|^{2}-\frac{\delta}{2\left(\theta+\lambda_{k}\right)}\|\phi\|^{2} \\
& +\|\phi\|-C_{3}\|w\|-C_{4},
\end{aligned}
$$

where $C_{3}=|\Omega|^{1 / 2} S K, C_{4}=C_{2}|\Omega|^{1 / 2}\left\|L_{2}\right\|_{2}+C_{2}|\Omega|$. Since

$$
\begin{aligned}
\left(1-\frac{\delta}{2\left(\theta+\lambda_{k}\right)}\|u\|\right)\|u\| \leq & \|w\|+\|\phi\| \\
& -\frac{\delta}{2\left(\theta+\lambda_{k}\right)}\left(\|\phi\|^{2}+\|w\|^{2}\right) \\
\leq & \left(1-\frac{\delta}{2\left(\theta+\lambda_{k}\right)}\|\phi\|\right)\|\phi\| \\
& +\|w\|,
\end{aligned}
$$

supposing $\delta \leq\left(\lambda_{k+m}-\lambda_{k}\right) / 2$, (87) becomes

$$
\begin{aligned}
J(w+\phi) \geq & \frac{\lambda_{k+m}-\left(\lambda_{k}+\delta\right)}{2\left(\theta+\lambda_{k+m}\right)}\|w\|^{2}-C_{3}\|w\|-C_{4} \\
& -\|w\|+\left(1-\frac{\delta}{2\left(\theta+\lambda_{k}\right)}\|u\|\right)\|u\| \\
\geq & \frac{\lambda_{k+m}-\lambda_{k}}{4\left(\theta+\lambda_{k+m}\right)}\|w\|^{2}-\left(C_{3}+1\right)\|w\| \\
& -C_{4}+\left(1-\frac{\delta}{2\left(\theta+\lambda_{k}\right)}\|u\|\right)\|u\|,
\end{aligned}
$$

since $\left(\lambda_{k+m}-\lambda_{k}\right) / 4\left(\theta+\lambda_{k+m}\right)>0$, so

$$
h(w)=\frac{\lambda_{k+m}-\lambda_{k}}{4\left(\theta+\lambda_{k+m}\right)}\|w\|^{2}-\left(C_{3}+1\right)\|w\|-C_{4}
$$

is bounded below for all $w \in W$; that is, there exists $C_{5} \in R$ such that $h(w) \geq C_{5}$; by (89) one gets

$$
\begin{aligned}
J(u) & \geq\left(1-\frac{\delta}{2\left(\theta+\lambda_{k}\right)}\|u\|\right)\|u\|+C_{5} \\
& =-\frac{\delta}{2\left(\theta+\lambda_{k}\right)}\|u\|^{2}+\|u\|+C_{5} .
\end{aligned}
$$

(ii) In case $\left(f_{4}\right)$, first we give some conclusions which are similar to Lemma 3 of [18]. Under the property of $F$, there exists a const $C$, and $G \in C(R, R)$ which is subadditive, that is,

$$
G(s+t) \leq G(s)+G(t)
$$

for all $s, t \in R$, and coercive, that is,

$$
G(s) \longrightarrow+\infty
$$

as $|s| \longrightarrow \infty$, and satisfies that

$$
G(s) \leq|s|+4
$$

for all $s \in R$, such that

$$
-F(x, s) \geq G(s)-C
$$

for all $s \in R$ and $x \in \bar{\Omega}$.

In fact, since $-F(x, s) \longrightarrow+\infty$ as $|s| \longrightarrow \infty$ uniformly for all $x \in \bar{\Omega}$, there exists a sequence of positive integers $n_{k}$ with $n_{k+1}>2 n_{k}$ for all positive integers $k$ such that

$$
-F(x, s) \geq k
$$

for all $|s| \geq n_{k}$ and all $x \in \bar{\Omega}$. Let $n_{0}=0$ and define

$$
G(s)=k+2+\frac{|s|-n_{k-1}}{n_{k}-n_{k-1}}
$$

for $n_{k-1} \leq|s|<n_{k}$, where $k \in N$.

By the definition of $G$ we have

$$
k+2 \leq G(s) \leq k+3
$$

for all $n_{k-1} \leq|s|<n_{k}$. By $\left(f_{4}\right)$ and $F \in C^{1}(\bar{\Omega} \times R, R)$, there exists $C_{F}>0$ such that

$$
-F(x, s) \geq-C_{F}, \quad \forall(x, s) \in\left(\Omega, R^{2}\right) .
$$

It follows that

$$
-F(x, s) \geq G(s)-C,
$$

where $C=C_{F}+4$. In fact, when $n_{k-1} \leq|s|<n_{k}$, for some $k \geq 2$, one has, by (96) and (98),

$$
-F(x, s) \geq k-1 \geq G(s)-4 \geq G(s)-C
$$

for all $x \in \bar{\Omega}$. When $|s|<n_{1}$, we have, by (98) and (99),

$$
-F(x, s) \geq-C_{F}=4-C \geq G(s)-C
$$

for all $x \in \bar{\Omega}$.

It is obvious that $G$ is continuous and coercive. Moreover, one has

$$
G(s) \leq|s|+4
$$

for all $s \in R$. In fact, for every $s \in R$, there exists $k \in N$ such that

$$
n_{k-1} \leq|s|<n_{k}
$$


which implies that

$$
G(s) \leq(k-1)+4 \leq n_{k-1}+4 \leq|s|+4
$$

for all $s \in R$ by (98) and the fact that $n_{k} \geq k$ for all integers $k \geq 0$.

Now we only need to prove the subadditivity of $G$. Let

$$
n_{k-1} \leq|s|<n_{k}, \quad n_{j-1} \leq|t|<n_{j}
$$

and $m=\max \{k, j\}$. Then we have

$$
|s+t| \leq|s|+|t|<n_{k}+n_{j} \leq 2 n_{m}<n_{m+1} .
$$

Hence we obtain, by (98),

$$
G(s+t) \leq m+4 \leq k+2+j+2 \leq G(s)+G(t),
$$

which shows that $G$ is subadditive.

For $u \in E_{k} \oplus W$, we assume that $u=w+\phi$, with $w \in W$ and $\phi \in E_{k}$; letting $0<\delta<\left(\lambda_{k+m}-\lambda_{k}\right) / 2$, by (28), (92), (94), and (95), one gets

$$
\begin{aligned}
J(w+\phi) \geq & \frac{\lambda_{k+m}-\left(\lambda_{k}+\delta\right)}{2\left(\theta+\lambda_{k+m}\right)}\|w\|^{2}-\frac{\delta}{2\left(\theta+\lambda_{k}\right)}\|\phi\|^{2} \\
& +\int_{\Omega} G(\phi+w) d x-C|\Omega| \\
\geq & \frac{\lambda_{k+m}-\left(\lambda_{k}+\delta\right)}{2\left(\theta+\lambda_{k+m}\right)}\|w\|^{2}-\frac{\delta}{2\left(\theta+\lambda_{k}\right)}\|\phi\|^{2} \\
& +\int_{\Omega} G(\phi) d x \\
& -\int_{\Omega} G(-w) d x-C|\Omega| \\
\geq & \frac{\lambda_{k+m}-\lambda_{k}}{4\left(\theta+\lambda_{k+m}\right)}\|w\|^{2}-\frac{\delta}{2\left(\theta+\lambda_{k}\right)}\|\phi\|^{2} \\
& +\int_{\Omega} G(\phi) d x \\
& -\int_{\Omega}(|w|+4) d x-C|\Omega| \\
\geq & \frac{\lambda_{k+m}-\lambda_{k}}{4\left(\theta+\lambda_{k+m}\right)}\|w\|^{2}-\frac{\delta}{2\left(\theta+\lambda_{k}\right)}\|z\|^{2} \\
& +\int_{\Omega} G(\phi) d x-S|\Omega|\|w\|-C_{1} \\
& g\left(\int_{\Omega} G(\phi) d x-\frac{\delta}{2\left(\theta+\lambda_{k}\right)}\|u\|^{2}\right. \\
& \\
& \\
&
\end{aligned}
$$

where $g(w)=\left(\left(\lambda_{k+m}-\lambda_{k}\right) / 4\left(\theta+\lambda_{k+m}\right)\right)\|w\|^{2}-S|\Omega|\|w\|-C_{1}$, $C_{1}=(4+C)|\Omega|$. Since $\phi \in E_{k}, E_{k}$ is a finite-dimensional subspace, and $G$ is coercive, from the proof of (75), one can get

$$
\lim _{\|\phi\| \rightarrow \infty} \int_{\Omega} G(\phi) d x=+\infty .
$$

That is, $\int_{\Omega} G(\phi) d x$ is coercive on $E_{k}$. Since $\left(\lambda_{k+m}-\lambda_{k}\right) / 4(\theta+$ $\left.\lambda_{k+m}\right)>0$, so $g(w)$ is coercive on $W$, and $\int_{\Omega} G(\phi) d x$ and $g(w)$ are bounded below, so it is obvious that

$$
\lim _{\|u\| \rightarrow \infty}\left(g(w)+\int_{\Omega} G(\phi) d x\right)=+\infty
$$

for all $u \in Z \oplus W$.

Considering (91), (109), and (111), we can choose $R_{2}$ large enough such that for all $\|u\| \geq R_{2}$ one gets

$$
g(w)+\int_{\Omega} G(\phi) d x>K_{V}+1
$$

(or $R_{2}+C_{5}>K_{V}+1$ for the case $\left(f_{3}\right)$ ) and property (52) holds; then for $0<\delta<\min \left\{2\left(\theta+\lambda_{k}\right) / R_{2}^{2},\left(\lambda_{k+m}-\lambda_{k}\right) / 2\right\}=\delta_{1}$ and $u \in R_{2} S_{Z W}$ one gets $J(u)>K_{V}$; that is, the property (51) holds.

\section{Proof of the Geometry in Propositions 9 and 10}

We finally give the proof of Propositions 9 and 10, which is nothing but a resume of the lemmata above, verifying that all the constants can be chosen sequentially without contradictions.

Proof of Proposition 9. Under hypotheses $\left(f_{0}\right)$ and $\left(f_{\infty}\right)$, if we fix a value $\lambda$, then we obtain the constant $D_{\lambda}$ from Lemma 11 and with this we get $\rho_{\lambda}$ from Lemma 12. If we consider also one of the two sets of hypotheses $\left(f_{1}\right)$ and $\left(f_{2}\right)$, then we proceed as follows. First of all, we determine (once forever) the constant $D_{W}$ from Lemma 11; with this we obtain from Lemma 14 the values $R_{1}$ and $\delta_{0}$. Then, for any (now fixed) $\lambda \in\left(\lambda_{k}-\delta_{0}, \lambda_{k}\right)$, we obtain from Lemma 11 the value $D_{\lambda}$. Finally, we can get from Lemma 12 the corresponding value of $\rho_{\lambda}>R_{1}$.

Proof of Proposition 10. Under hypotheses $\left(f_{0}\right)$ and $\left(f_{\infty}\right)$, if we fix a value $\lambda \in\left(\lambda_{k}, \lambda_{k+m}\right)$, then we obtain the constant $K_{\lambda}$ from Lemma 11 and with this we get $\beta_{\lambda}$ from Lemma 12. If we consider also one of the two sets of hypotheses $\left(f_{3}\right)$ and $\left(f_{4}\right)$, then we proceed as follows. First of all, we determine (once forever) the constant $K_{V}$ from Lemma 12; with this we obtain from Lemma 15 the values $R_{2}$ and $\delta_{1}$. Since we have $R_{2}$, we can get from Lemma 11 the constant $E$ and with this obtain $\xi$ from Lemma 12.

Finally, for any (now fixed) $\lambda \in\left(\lambda_{k}, \lambda_{k}+\delta_{1}\right)$, we obtain from Lemma 11 the constant $K_{\lambda}$ and with this we get from Lemma 12 the corresponding value of $\beta_{\lambda}>R_{2}$.

\section{Conflict of Interests}

The authors declare that there is no conflict of interests regarding the publication of this paper.

\section{Acknowledgment}

This work was supported by the Science and Technology Foundation of Guizhou Province (no. LKB[2012]19; no. [2013]2141). 


\section{References}

[1] C. Li, "The existence of infinitely many solutions of a class of nonlinear elliptic equations with Neumann boundary condition for both resonance and oscillation problems," Nonlinear Analysis: Theory, Methods \& Applications, vol. 54, no. 3, pp. 431-443, 2003.

[2] A. Qian, "Existence of infinitely many nodal solutions for a superlinear Neumann boundary value problem," Boundary Value Problems, vol. 2005, no. 3, pp. 329-335, 2005.

[3] D. Motreanu, D. O’Regan, and N. S. Papageorgiou, "A unified treatment using critical point methods of the existence of multiple solutions for superlinear and sublinear Neumann problems," Communications on Pure and Applied Analysis, vol. 10, no. 6, pp. 1791-1816, 2011.

[4] C.-L. Tang and X.-P. Wu, "Existence and multiplicity for solutions of Neumann problem for semilinear elliptic equations," Journal of Mathematical Analysis and Applications, vol. 288, no. 2, pp. 660-670, 2003.

[5] D. Motreanu, V. V. Motreanu, and N. S. Papageorgiou, Topological and Variational Methods with Applications to Nonlinear Boundary Value Problems, Springer, New York, NY, USA, 2014.

[6] L. Gasiński and N. S. Papageorgiou, "Multiplicity of solutions for Neumann problems with an indefinite and unbounded potential," Communications on Pure and Applied Analysis, vol. 12, no. 5, pp. 1985-1999, 2013.

[7] N. S. Papageorgiou and V. D. Rădulescu, "Semilinear Neumann problems with indefinite and unbounded potential and crossing nonlinearity," in Recent trends in Nonlinear Partial Differential Equations. II. Stationary Problems, vol. 595 of Contemporary Mathematics, pp. 293-315, American Mathematical Society, Providence, RI, USA, 2013.

[8] J. Mawhin and K. Schmitt, "Nonlinear eigenvalue problems with the parameter near resonance," Annales Polonici Mathematici, vol. 51, pp. 241-248, 1990.

[9] T. F. Ma, M. Ramos, and L. Sanchez, "Multiple solutions for a class of nonlinear boundary value problems near resonance: a variational approach," Nonlinear Analysis: Theory, Methods \& Applications, vol. 30, no. 6, pp. 3301-3311, 1997.

[10] T. F. Ma and M. L. Pelicer, "Perturbations near resonance for the p-laplacian in $\mathbb{R}^{N}$," Abstract and Applied Analysis, vol. 7, no. 6, pp. 323-334, 2002.

[11] T. F. Ma and L. Sanchez, "Three solutions of a quasilinear elliptic problem near resonance," Mathematica Slovaca, vol. 47, no. 4, pp. 451-457, 1997.

[12] Z.-Q. Ou and C.-L. Tang, "Existence and multiplicity results for some elliptic systems at resonance," Nonlinear Analysis: Theory, Methods \& Applications, vol. 71, no. 7-8, pp. 2660-2666, 2009.

[13] F. O. de Paiva and E. Massa, "Semilinear elliptic problems near resonance with a nonprincipal eigenvalue," Journal of Mathematical Analysis and Applications, vol. 342, no. 1, pp. 638650, 2008.

[14] H.-M. Suo and C.-L. Tang, "Degenerate semilinear elliptic problems near resonance with a nonprincipal eigenvalue," Bulletin of the Korean Mathematical Society, vol. 49, no. 4, pp. 669-684, 2012.

[15] L. C. Evance, Partial Differential Equations, Graduate Studies in Mathematics, vol. 19, American Mathematical Society, Providence, RI, USA, 1998.

[16] A. Marino, A. M. Micheletti, and A. Pistoia, "A nonsymmetric asymptotically linear elliptic problem," Topological Methods in Nonlinear Analysis, vol. 4, no. 2, pp. 289-339, 1994.
[17] P. H. Rabinowitz, Minimax Methods in Critical Point Theory with Applications to Differential Equations, vol. 65 of CBMS Regional Conference Series in Mathematics, American Mathematical Society, Providence, RI, USA, 1986.

[18] C.-L. Tang and X.-P. Wu, "Periodic solutions for second order systems with not uniformly coercive potential," Journal of Mathematical Analysis and Applications, vol. 259, no. 2, pp. 386397, 2001. 


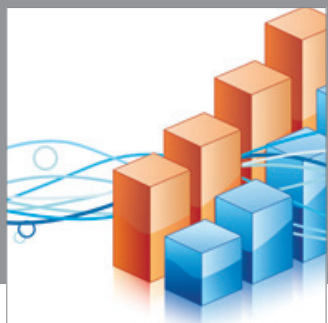

Advances in

Operations Research

mansans

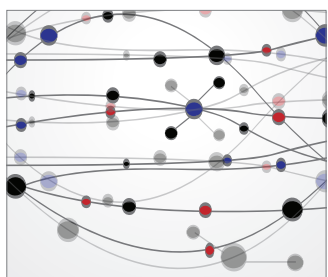

The Scientific World Journal
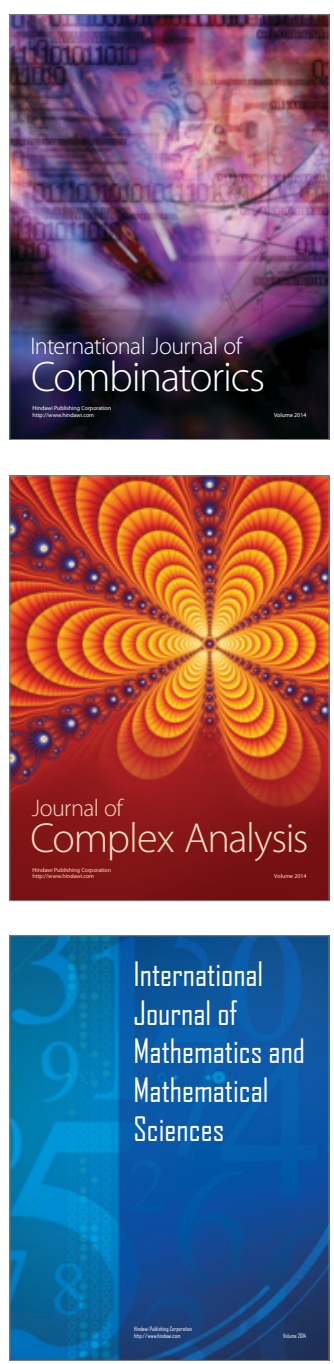
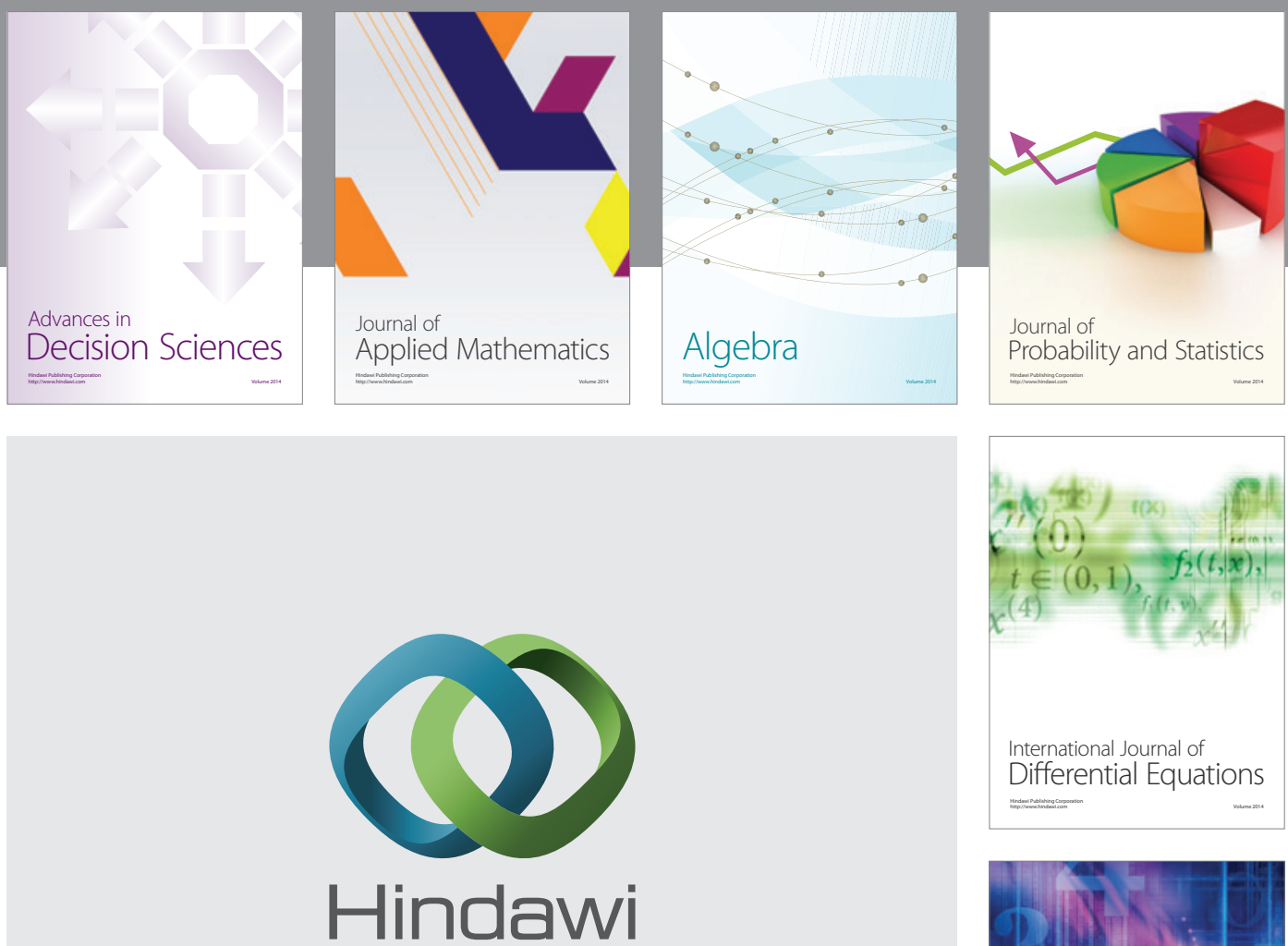

Submit your manuscripts at http://www.hindawi.com
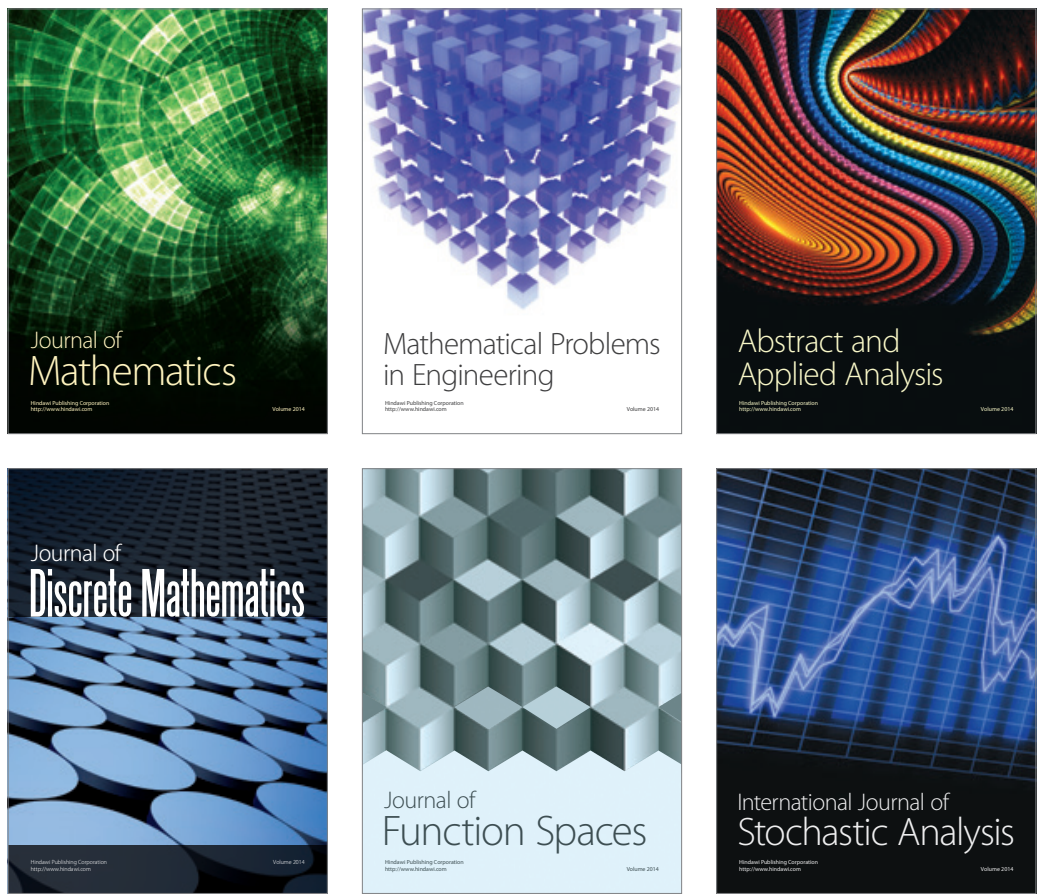

Journal of

Function Spaces

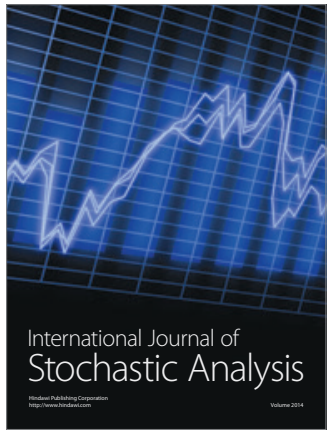

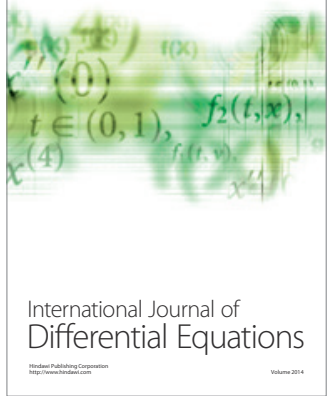
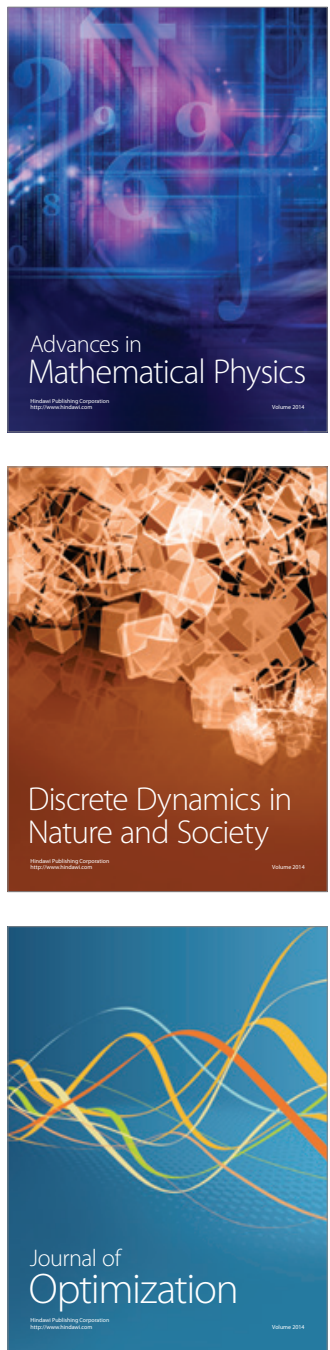